\title{
IN HAL'T
}

Seite

Aufruf der Konferenz der asiatisch-afrikanischen Völker an die Gelehrten der ganzen Welt. . . . . . . . 145

J. Znosкo: Die Aussichten der Erkundung von Eisenerzlagerstätten im Vesulien von Lęczyca . . . . . . 146

Borchert \& CISSARz: Die Erzlagerstätten des initialen Magmatismus, referiert von Thomas KaEmmel und ERich Knauer . . . . . . . . . . . . . . . . 149

E. v. Hoyningen-Hunne: Gedanken über die Nutzung von Baustoffvorkommen im Deckgebirge der Braunkohle . . . . . . . . . . . . . . . 152

A. D. Kalenow: Zur Einschätzung der Gehalte und der Vorräte beigemengter Elemente. . . . . . . . . 155

F. STAMmberger: Einige Bemerkungen zur Diskussion über die Vorratsklassifikation . . . . . . . 157
T. NöTzold: Die Möglichkeiten makropaläobotanischer Untersuchungen zur Erkundung der Braunkohle . . 161

F. REUTER: Die Wasserdurchlässigkeitsprüfungen und Baugrundvergütung als ingenieurgeologische Untersuchungsmethoden bei Talsperrenbauten. . . . . 166

M. Rost: Zu Fragen der ingenieurgeologischen Baugrundkartierung, besonders im Mittelgebirge . . . . 174

F. A. TREBIN : Die Erdölindustrie Burmas, referiert von H. Schlegel . . . . . . . . . . . . . . . . . 179

H. REH: Dem Andenken des großen Prospektors Hans Merensky . . . . . . . . . . . . . . . . . . 182

Lesesteine . . . . . . . . . . . . . . . . . 186

Besprechungen und Referate . . . . . . . . . . 187

Nachrichten und Informationen . . . . . . . 195

Die Zeitschrift Für ANGEWANDtE Geologie berichtet ständig ausführlich über folgende Arbeitsgebiete: Geologische Grundlagenforschung und Lagerstättenforschung / Methodik der geologisehen Errkundung / Ökonomie und Planung der geologischen Erkundung / Technik der geologischen Erkundung / Geologie und Lagerstättenkunde im Ausland / Bibliographie, Verordnungen, Richtlinien, Konferenzen, Personalnachrichten

Dem Redaktionskollegium gehören an:

Prof. Dipl.-Berging. BÜHRIG, Nordhausen - Dr. HECK, Schwerin - Dr. JUBELT, Halle - Prof. Dr. KAUTZSCH, Berlin

Prof. Dr. LANGE, Berlin - Dr. MEINHOLD, Leipzig - Dr. NOSSKE, Leipzig - Prof. Dr. PIETZSCH, Freiberg

Dr. REH, Jena - Prof. Dr. SCHÜLLER, Berlin - Dipl.-Berging.-Geologe STAMMBERGER, Berlin

Dr. STOCK, Berlin - Prof. Dr. WATZNAUER, Karl-Marx-Stadt

Chefredakteur: Prof. Dr. ERICH JANGE, Berlin

Die Zeitschrifr Für ANGewandte Geologie ist kein Organ einer engen Fachgruppe. Auf ihren Seiten können alle strittigen Fragen der praktischen Geologie behandelt werden. Die Autoren übernehmen für ihr Aufsätze die übliche Verantwortung.

Herausgeber: Staatliche Geologische Kommission und Zentrale Vorratskommission für mineralische Rohstoffe der Deutschen Demokratischen Republik. Chefredakteur: Prof. Dr. Erich Lange, Berlin. Redaktion: Berlin N 4, InvalidenstraBe 44. Verlag: Akademle-Verlag GmbH, Berlin W 8, MohrenstraBe 39 (Fernsprecher 200386, Postscheckkonto: Berlin 35021). Bestell- und Verlagsnummer des Heftes: 1047/4/4. Die "Zeitschrift für angewandte Geologie“ erscheint monatlich. Bezugspreis 2,- DM je Heft, Doppelheft 4,-DM. - Satz und Druck: Druckhaus „Maxim Gorki", Altenburg. Veröffentlicht unter der Lizenznummer ZLN 5008 des Ministeriums für Kultur, Hauptverwaltung Verlagswesen. Karten: MdI. der DDR Nr. 3871,3913, 3999 / K 11. Printed in Germany. 